Communications in Physics, Vol.18, No.1 (2008), pp. 19-22

\title{
ON GENERALIZED q-DEFORMED FERMIONIC OSCILLATORS
}

\author{
LUU THI KIM THANH \\ Hanoi Pedagogical University No. 2
}

\begin{abstract}
In this work we consider a version of generalized q-deformed fermionic oscillators and some their applications. The corresponding coherent states are constructed. The statistical distribution of generalized q-deformed fermionic oscillators system, expressions for quadrature variances and the average particle number in the coherent states are calculated.
\end{abstract}

\section{INTRODUCTION}

Quantum algebras (also called quantum groups) are deformed versions of the usual Lie algebras, to which they reduce when the deformation parameter is set equal to unity. Their use in physics became popular with the introduction of the q-deformed harmonic oscillator [1] as a tool for providing a boson realization of the quantum algebra $\mathrm{SU}_{q}(2)$. Initially used for solving the quantum Yang-Baxter equation [2], quantum algebras have subsequently found applications in several branches of physics, as, for example, in the description of spin chains, anyones, quantum optics [3] and in conformal field theories. By now several kinds of generalized deformed oscillators and generallized algebras [4] have been introduced.

The formalism of coherent states in an important part of quantum theory. Coherent states play a special role not only in optics but also in condensed matter physics and particle physics. They describle physically realizable system such as light emitted from a laser operating for above thresshold or Bose-Einstein condensated excitons/biexciton, etc..

A good deal of work has been devoted to the study of coherent states for bosons and fermions. During the last few years the concept of coherent states has been extended to the q-deformed oscillators [5].

The aim of this work is to study systematically the concept of generalized q-deformed fermionic oscillators and their coherent states. The obtained results include on an equal footing the usual q-deformed fermionic oscillators and the "quons" of infinite statistics. The contents of the paper are arranged as follows. In Sec. II we consider the generalized q-deformed single mode fermionic oscillators. In Sec. III we are dealing with their statistics distribution. Sec. IV is devoted to generalized q-deformed coherent states.

\section{THE GENERALIZED $q$-DEFORMED FERMIONIC OSCILLATORS}

We consider the fermionic oscillators with the deformed commutation rule of the form:

$$
b b^{+}+q b^{+} b=q^{c N}
$$


where $N$ is oscillator number operator

$$
\begin{aligned}
& {[N, b]=-b ;} \\
& {\left[N, b^{+}\right]=b,}
\end{aligned}
$$

$q$ and $c$ are some parameters.

Equation (1) gives:

$$
b b^{+n}=(-1)^{n} q^{n} b^{+n} b+\{n\}_{q}^{(c)}\left(b^{+}\right)^{n-1} q^{c N}
$$

where the general notation

is used.

$$
\{n\}_{q}^{(c)}=\frac{q^{c n}+(-1)^{n+1} q^{n}}{q^{c}+q}
$$

It is seen from the equation (3) that the algebra (1) can be realised in the Fock space spanned by the orthonormalise eigenstates of the operator $N$,

$$
|n\rangle=\frac{1}{\sqrt{\{n\}_{q}^{(c)} !}} b^{+n}|0\rangle
$$

and in this space the following relations hold:

$$
b^{+} b=\{N\}_{q}^{(c)} ; b b^{+}=\{N+1\}_{q}^{(c)} .
$$

\section{THE STATISTICAL DISTRIBUTION}

The statistical distribution of the operator $F$ is defined through the formula

$$
\langle F\rangle=\frac{1}{Z} \operatorname{Tr}\left(e^{-\beta H} F\right)
$$

where $Z$ is the partition function

$$
Z \equiv \operatorname{Tr}\left(e^{-\beta H}\right)
$$

which determines the themodynamic properties of the system, $\beta=\frac{1}{k T}, H$ is Hamiltonian, which is usually taken of the form $H=(\varepsilon-\mu) N, \varepsilon$ being one particle oscillator energy, $\mu$ is chemical potential. The trace must be taken over a complete set of states.

The calculations give the following results:

$$
\left\langle b^{+} b\right\rangle=\frac{e^{\beta(\varepsilon-\mu)}-1}{e^{2 \beta(\varepsilon-\mu)}+\left(q-q^{c}\right) e^{\beta(\varepsilon-\mu)}-q^{1-c}} .
$$

From this we recover the familiar formula of Fermi-Dirac statistical distribution

$$
\left\langle b^{+} b\right\rangle=\frac{1}{e^{\beta(\varepsilon-\mu)}+1}
$$

where $q=1$, and the result

$$
\left\langle b^{+} b\right\rangle=\frac{e^{\beta(\varepsilon-\mu)}}{e^{2 \beta(\varepsilon-\mu)}+\left(q-q^{-1}\right) e^{\beta(\varepsilon-\mu)}+1} .
$$

for usual q-deformed statistical distribution when $c=1$. 
In the limit of "infinite statistics", $c=0, q=0$, we have

$$
\left\langle b^{+} b\right\rangle=e^{-\beta(\varepsilon-\mu)} \text {. }
$$

\section{GENERALIZED q-DEFORMED COHERENT STATES}

Unlike Fock states, in which the particle number is definite while the phase is completely random, coherent states have small phase fluctuation but contain an uncertain particle number. For this reason coherent states can be mathematically viewed as eigenstates of the oscillator annihilation operator

$$
\begin{aligned}
b|z\rangle & =z|z\rangle ; \\
\langle z| b^{+} & =z^{*}\langle z|
\end{aligned}
$$

and it then follows from (3) that:

$$
\left\langle z\left|=c(z) \sum_{n=0}^{\infty} \frac{z^{n}\left(b^{+}\right)^{n}|0\rangle}{\{n\}_{q}^{(c)} !}=c(z) \sum_{n=0}^{\infty} \frac{z^{n}}{\sqrt{\{n\}_{q}^{(c)} !}}\right| n\right\rangle
$$

where $c(z)$ is the normalization constant,

$$
c(z)=\frac{1}{\sqrt{\{e\}^{|z|^{2}}}}
$$

and $\{e\}^{x}$ is the "Generalized $q$-deformed exponential" function defined by formula

$$
\{e\}^{x}=\sum_{n=0}^{\infty} \frac{x^{n}}{\{n\}_{q}^{(c)}}
$$

Equation (1) leads to the commutation relation

$$
[P, Q]=i \hbar\left(\{N\}_{q}^{(c)}-\{N+1\}_{q}^{(c)}\right)
$$

between cordinate and momentum operators defined as

$$
\begin{aligned}
& Q=\left(\frac{\hbar}{2 m \omega}\right)^{1 / 2}\left(b^{+}+b\right) ; \\
& P=i\left(\frac{m \hbar \omega}{2}\right)^{1 / 2}\left(b^{+}-b\right)
\end{aligned}
$$

with $m$ and $\omega$ being mass and frequency of the oscillator.

Instead of (15) the following dimensionless quantities are often used:

$$
\begin{aligned}
& \tilde{Q}=\left(\frac{m \omega}{2 \hbar}\right)^{1 / 2} Q=\frac{1}{2}\left(b^{+}+b\right) ; \\
& \tilde{P}=(2 m \hbar \omega)^{1 / 2} P=\frac{i}{2}\left(b^{+}-b\right) .
\end{aligned}
$$

For the quadrature variances the calculations give the following result

$$
\left\langle z\left|(\Delta \widetilde{Q})^{2}\right| z\right\rangle=\left\langle z\left|(\Delta \widetilde{P})^{2}\right| z\right\rangle=\frac{1}{4} F
$$


where

and

$$
F=\{e\}^{\left(q^{c}-1\right)|z|^{2}}-(q+1)|z|^{2}
$$

$$
\left\langle z\left|(\Delta \widetilde{Q})^{2}\right|\right\rangle \cdot\left\langle z\left|(\Delta \widetilde{P})^{2}\right| z\right\rangle=\frac{1}{16} F^{2}
$$

The average partial number in coherent state and probability for this states to be in the $n$ - particle state are:

$$
\begin{gathered}
\langle z|N| z\rangle=|z|^{2}\left(\{e\}^{|z|^{2}}\right)^{-1} \cdot \sum_{n=0}^{\infty} \frac{n+1}{\{n+1\}^{(c)}}|z|^{2 n} \\
W_{n}=|<n| z>\left.\right|^{2}=\left(\{e\}^{|z|^{2}}\right)^{-1} \frac{z^{2 n}}{\{n\}_{q}^{(c)} !}
\end{gathered}
$$

The results recover familiar formulae when deformation parameters tend to particular value.

\section{ACKNOWLEDGMENT}

We would like to thank prof. Dao Vong Duc and Prof. Tran Huu Phat for useful discussions.

\section{REFERENCES}

[1] A. J. Macfarlane, J. Phys. A, 22 (1989) 4581.

[2] M. Jimbo, Yang Baxter Equation in Integrable Systems, Adv. Series in Math. Phys. 10 (Wold Scientific, Singapore, 1990).

[3] K. P. Marzlin, Int. J. Theor. Phys., 26 (1997) 841.

[4] Dao Vong Duc, Pro. Inter. Conf. On Frontiers in Quantum Physics, Kuala lumpur 1998, SpingerVerlag, p. 272.

[5] Luu Thi Kim Thanh, Comm. in Phys., 17 (2007), 9-12.

Received 18 November $200 \%$. 
